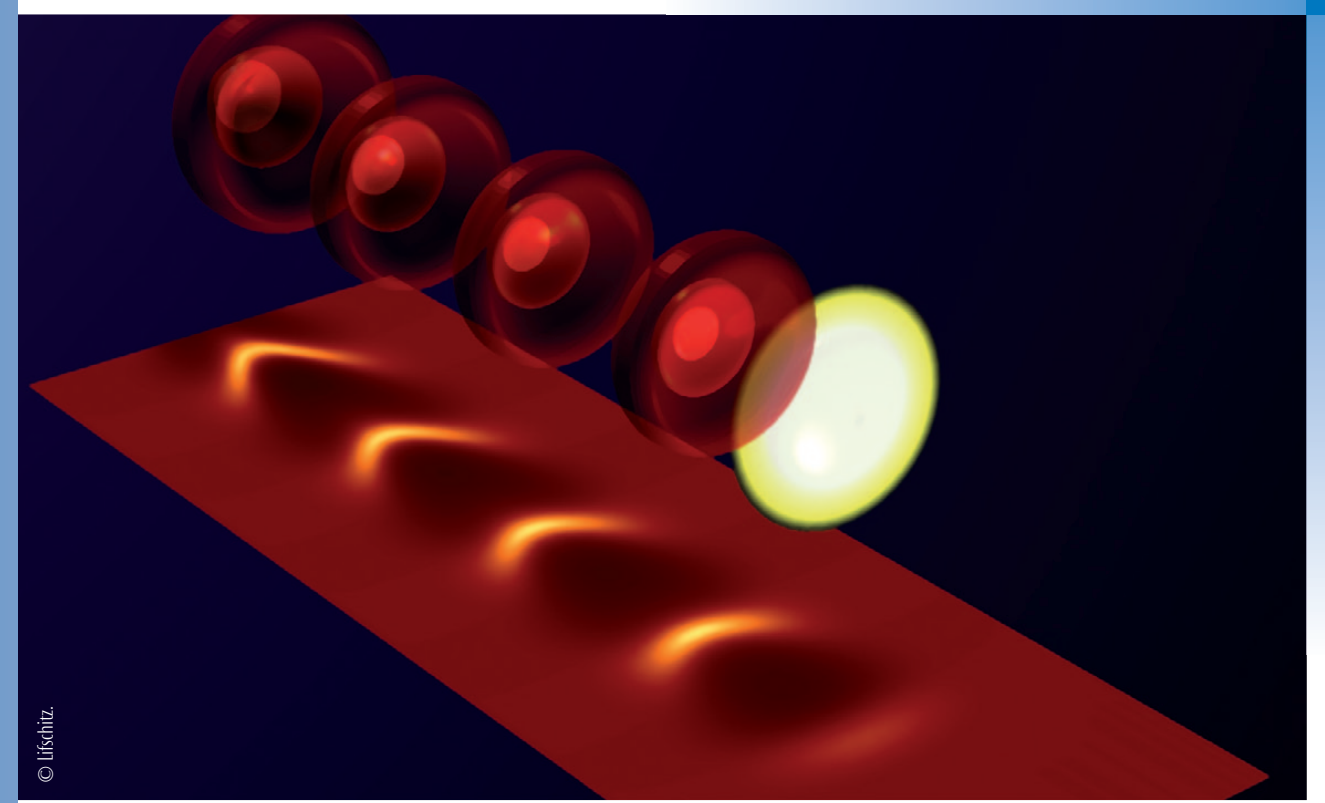

Principe du sillage laser (simulation numérique) : l'impulsion laser (en jaune) excite dans son sillage l'onde plasma relativiste.

\title{
Accélérateurs à plasma laser : principes et applications
}

Victor Malka (victor.malka@ensta-paristech.fr), Cédric Thaury, Sébastien Corde,

Kim Ta Phuoc et Antoine Rousse

Laboratoire d'optique appliquée, unité mixte de recherche du CNRS, de l'École Nationale Supérieure de Techniques Avancées et de l'École polytechnique, 91761 Palaiseau Cedex.

En transmettant, à des faisceaux d'électrons ou à des faisceaux de rayons $X$, les propriétés des impulsions lasers, les physiciens sont capables aujourd'hui de réaliser des accélérateurs à plasma compacts et compétitifs, délivrant des faisceaux de particules ou de rayonnement $X$ très brillants.

Pour réaliser cette performance, il faut, d'une part, mettre en mouvement de façon collective les électrons du plasma, et, d'autre part, contrôler dans un très petit volume l'injection des particules à accélérer.

\section{Les accélérateurs dits conventionnels}

Les accélérateurs d'électrons sont utilisés dans de nombreux domaines, allant de la médecine à la physique des particules, en passant par la radiobiologie ou la physique de la matière condensée. Le marché annuel des accélérateurs industriels est évalué à quelques milliards d'euros. Les accélérateurs à usage fondamental pour la physique des hautes énergies et pour les sources de rayonnement $\mathrm{X}$ de $3^{\mathrm{e}}$ et $4^{\mathrm{e}}$ générations (synchrotrons et lasers à électrons libres) représentent un investissement du même ordre de grandeur.

Les accélérateurs modernes utilisent des ondes électromagnétiques radiofréquence (RF) pour accélérer des particules chargées à des vitesses relativistes. Développée depuis plus d'un demi-siècle, cette technologie a fait ses preuves : elle permet de produire de façon stable et robuste des faisceaux de particules d'excellente qualité. Dans un accélérateur, l'énergie des particules est proportionnelle au produit du champ électrique et de la distance sur laquelle on l'applique. Or, le champ accélérateur dans ces cavités RF est limité à quelques dizaines de mégavolts par mètre : au-delà, les parois de la structure, ionisées par le champ électrique, commencent à être endommagées (phénomène de claquage). Cette limite du champ explique pourquoi les accélérateurs deviennent de plus en plus grands. Par exemple, le Grand Collisionneur de Hadrons (LHC) du CERN, près de Genève, qui mène avec succès sa quête du boson de Higgs [1], est un accélérateur circulaire aussi long que le boulevard périphérique de Paris. Son possible successeur pourrait être l'International Linear Collider (ILC), qui utilisera 16000 cavités RF pour accélérer des électrons et des positrons à 250 gigaélectron-volts $(\mathrm{GeV})$ sur $31 \mathrm{~km}$, ou le Compact Linear Collider (CLIC), d'une longueur de $40 \mathrm{~km}$, qui fera des collisions électrons-positrons à 3 téraélectron-volts $(\mathrm{TeV})$.

La longueur des accélérateurs ne pouvant croître indéfiniment, on comprend toute l'importance de chercher des solutions alternatives compactes à l'accélération de particules. 


\section{\>}

\section{Les accélérateurs à plasma laser : des premiers petits pas au pas de géant}

Sur la base de simulations numériques et de modèles théoriques, $T$. Tajima et J. Dawson suggéraient à la fin des années 1970 que, dans un plasma, des électrons pourraient être accélérés à une vitesse proche de celle de la lumière. Milieux ionisés, constitués d'électrons libres et d'ions, les plasmas peuvent, par nature, supporter des champs électriques mille à dix mille fois supérieurs à ceux utilisés dans les accélérateurs traditionnels ! Dans un plasma, la distance sur laquelle une particule doit être accélérée pour parvenir à une énergie donnée est donc considérablement réduite par rapport aux machines actuelles. Au début des années 1990, la confirmation expérimentale a été apportée par une équipe américaine qui est parvenue, pour la première fois, à accélérer des électrons injectés dans un plasma. En 1995, en Grande-Bretagne, des faisceaux d'électrons de grande énergie, allant jusqu'à 44 mégaélectron-volts $(\mathrm{MeV})$, ont été directement produits par une équipe anglo-francoaméricaine, sans qu'une source extérieure de particules soit nécessaire. Quelques années plus tard, des résultats analogues ont été obtenus avec des lasers tout aussi puissants, mais d'une énergie et d'une taille beaucoup plus modestes, comme celui du Laboratoire d'optique appliquée (LOA) à Palaiseau. Dès 1999, la production de faisceaux d'électrons de $70 \mathrm{MeV}$ a été démontrée avec ce laser compact. Puis en 2002, dans des ondes plasmas de très fortes amplitudes excitées dans le régime de sillage forcé, des faisceaux d'électrons d'énergie encore plus élevée (200 MeV) ont été obtenus [2]. En améliorant ce régime, une avancée notable a été réalisée en 2004 où, en même temps que deux autres équipes, aux États-Unis et en Grande-Bretagne, nous avons obtenu pour la première fois des faisceaux qui, par leurs qualités, s'approchaient de ceux délivrés par les machines traditionnelles, mais après une distance d'accélération de quelques millimètres seulement [3]. Plus récemment, en 2006, nous avons encore amélioré notre dispositif en éclairant le plasma avec deux impulsions laser : ce nouveau schéma d'accélérateur produit alors un faisceau d'électrons stable, et dont la charge et l'énergie peuvent être précisément contrôlées en jouant simplement sur les paramètres des impulsions laser [4].
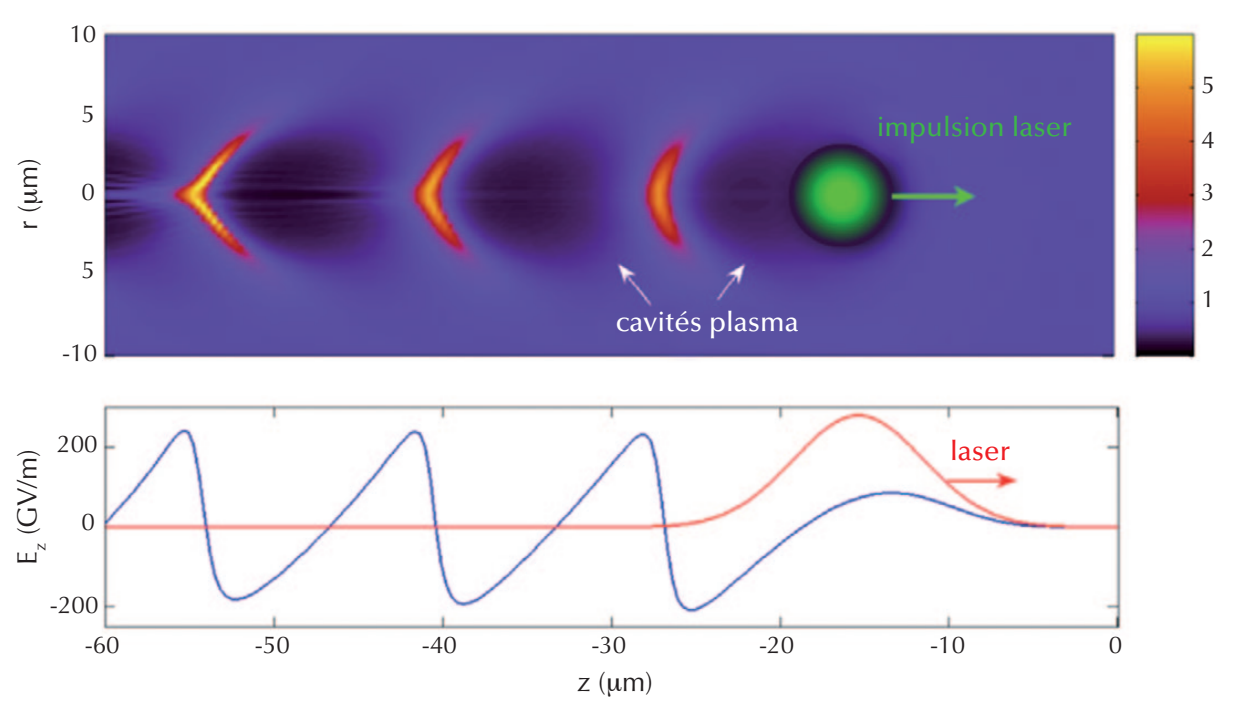

1. Onde plasma. L'impulsion laser (en vert) crée une perturbation de densité électronique (figure du haut). Le profil du champ électrique associé à cette onde plasma relativiste est illustré sur la figure du bas.

Une brièveté de l'ordre de la femtoseconde [5], un courant crête de quelques $\mathrm{kA}$, une dispersion en énergie de l'ordre du pourcent, et des énergies atteintes qui dépassent aujourd'hui le $\mathrm{GeV}$, représentent les paramètres actuels des accélérateurs à plasma laser.

\section{Comment ça marche ?}

En focalisant une impulsion laser de forte puissance sur une cible gazeuse, le champ électrique gigantesque qui lui est associé arrache les électrons aux atomes constituant le gaz; celui-ci passe alors immédiatement de son état initial gazeux à celui de plasma. Dans le plasma ainsi formé, la force pondéromotrice $^{(1)}$, qui est proportionnelle au gradient de l'intensité laser, agit comme une force de pression de radiation qui chasse les électrons hors du chemin de l'impulsion lumineuse. Plus lourds, les ions sont peu sensibles à cette pression, et restent immobiles pendant la durée du phénomène. La différence de répartition des charges électroniques et ioniques crée un champ électrique qui tend à ramener les électrons vers leurs positions d'origine. Les électrons se mettent alors à osciller vers l'avant puis vers l'arrière, avec une période directement liée à la densité initiale du milieu. Cette oscillation collective est appelée " onde plasma ", et le champ électrique associé à ce mouvement constitue l'onde propagative nécessaire à l'accélération des électrons. Le schéma de principe est illustré sur la figure 1. Si la durée de l'impulsion laser est de l'ordre de la période d'oscillation du plasma, le transfert d'énergie du laser vers l'onde plasma est optimal. Ce phénomène porte le nom de "sillage laser ", par similitude avec un bateau qui crée une vague dans son sillage. Si cette vague (l'onde plasma) est d'une amplitude suffisante, des surfeurs (les électrons) peuvent s'en servir pour gagner de la vitesse. Ainsi, dans nos expériences, nous produisons de gigantesques « vagues » derrière l'impulsion laser, et nous nous en servons pour accélérer les électrons [6].

Une fois la structure accélératrice établie, il convient d'y injecter les électrons de façon externe avec un faisceau issu d'un accélérateur, ou de façon interne en piégeant certains des électrons du plasma. Les dimensions radiale et longitudinale de la structure accélératrice étant très petites, de l'ordre de la dizaine de microns, le défi à relever était de taille. Au cours des premières expériences, les ondes étaient amplifiées jusqu'à atteindre le déferlement. Les électrons étaient accélérés de façon plus ou moins aléatoire, et les faisceaux obtenus étaient de mauvaise qualité : les électrons se trouvaient animés de vitesses différentes, et accélérés dans des directions différentes.

Les résultats publiés dans Nature en 2004 [3] ont montré qu'en adaptant la densité électronique et la durée de l'impulsion laser ${ }^{(2)}$, un faisceau laser suffisamment intense pouvait créer une bulle plasma 

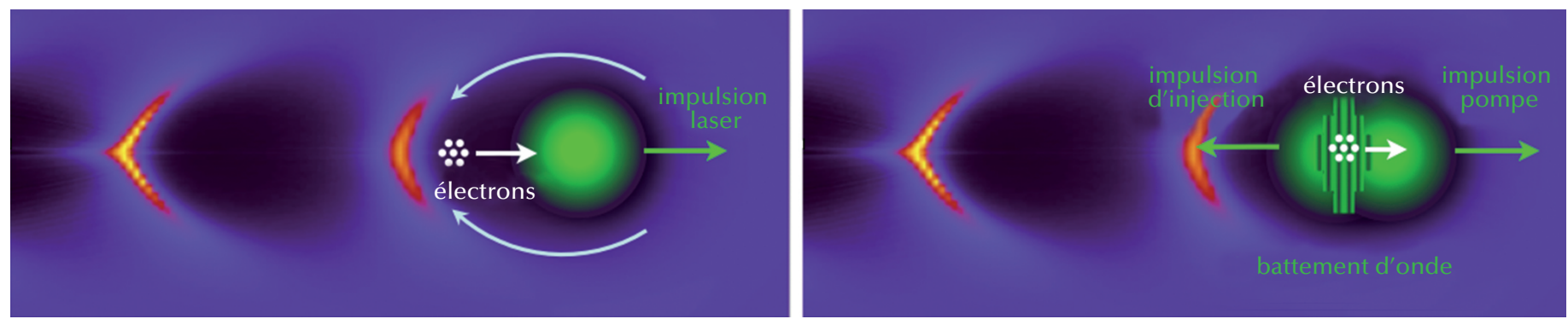

2. À gauche, le régime de la bulle. L'impulsion laser (en vert) chasse les électrons de son passage, créant une bulle ionique ; les électrons circulent autour de la bulle avant d'être injectés sur l'axe du laser. La charge du paquet d'électrons croît jusqu'à atteindre la densité du plasma, le faisceau d'électrons écrante le champ de la bulle et l'injection s'arrête. Cette double localisation temporelle et spatiale de l'injection permet l'obtention de faisceaux d'électrons quasi monoénergétiques.

À droite, le régime de collisions d'impulsions laser. L'impulsion d'injection entre en collision frontale avec l'impulsion pompe qui a créé l'onde de sillage. Au cours de la collision, le chauffage de certains électrons dû au battement des deux ondes électromagnétiques associées à ces deux impulsions laser, permet l'injection ultérieure de ces électrons qui ont acquis une énergie suffisante pour être piégés.

capable de piéger localement des électrons (fig. 2 à gauche). Le caractère aléatoire du déferlement peut ainsi être évité : les électrons étant tous piégés au même endroit, ils acquièrent tous la même énergie lors de l'accélération.

En 2006, en réalisant l'injection par collision de deux impulsions laser (de même longueur d'onde), nous avons montré un contrôle encore plus précis des paramètres du faisceau d'électrons. Ici, une première impulsion laser (dite impulsion pompe) excite l'onde de sillage, puis une seconde impulsion laser (dite impulsion d'injection) qui se propage en sens inverse, entre en collision avec l'impulsion principale et chauffe très localement certains électrons du plasma au moment où les deux impulsions laser se recouvrent (fig. 2 à droite). Certains de ces électrons se trouvent alors injectés et accélérés dans l'onde de sillage, produisant ainsi un faisceau d'électrons de bien meilleure qualité. Plus important encore, en jouant de façon très simple sur les paramètres de ce deuxième faisceau laser - délai avec l'impulsion pompe, intensité ou polarisation - il est possible de contrôler la charge, l'énergie et la dispersion en énergie du faisceau de particules. Les ondes créées étant en outre de plus faible amplitude - car il n'est plus nécessaire désormais qu'elles déferlent spontanément le processus d'injection est mieux maitrisé et le faisceau produit est très reproductible.

D'autres schémas d'injection, utilisant des gradients de densité ou des mélanges gazeux ont aussi été explorés récemment ; ils montrent toutes les possibilités d'études et d'évolutions de ce domaine de recherche.

\section{Des faisceaux $X$ très brillants}

Les oscillations des électrons relativistes, soit dans le champ électrique radial présent dans les accélérateurs à plasma laser, soit dans le champ électromagnétique du laser, ont permis de produire des flashs $\mathrm{X}$ très intenses par rayonnement "bêtatron " dans le premier cas, et par rayonnement «Compton» dans le second cas.

\section{Rayonnement bêtatron}

La figure 3 montre le principe d'émission du rayonnement bêtatron. Au sein de la structure accélératrice, les électrons éjectés sous l'effet de la force pondéromotrice du laser se concentrent sur la surface d'une sphère, laissant son centre rempli d'ions. Le champ électrique longitudinal accélère les électrons vers l'avant, tandis que le champ

électrique radial renvoie les électrons qui sont injectés hors de l'axe vers le centre de la cavité (fig. 3). Ces électrons oscillent donc autour de l'axe de propagation de l'impulsion laser. La longueur d'onde des oscillations est de l'ordre de la centaine de microns, et l'amplitude transverse de l'ordre du micron. On appelle ces oscillations les " oscillations bêtatron ", ce qui a donné son nom à la source. Ce mouvement relativiste est responsable de l'émission de faisceaux de rayonnement. Il s'agit de rayonnement de charges en mouvement relativiste, directement comparable au rayonnement synchrotron. Les faisceaux de rayonnement bêtatron produits actuellement sont peu divergents (environ 1 degré), contiennent des photons ayant une énergie de plusieurs dizaines de $\mathrm{keV}$ [7], et ont des brillances crête équivalentes à celles des synchrotrons.

\〉

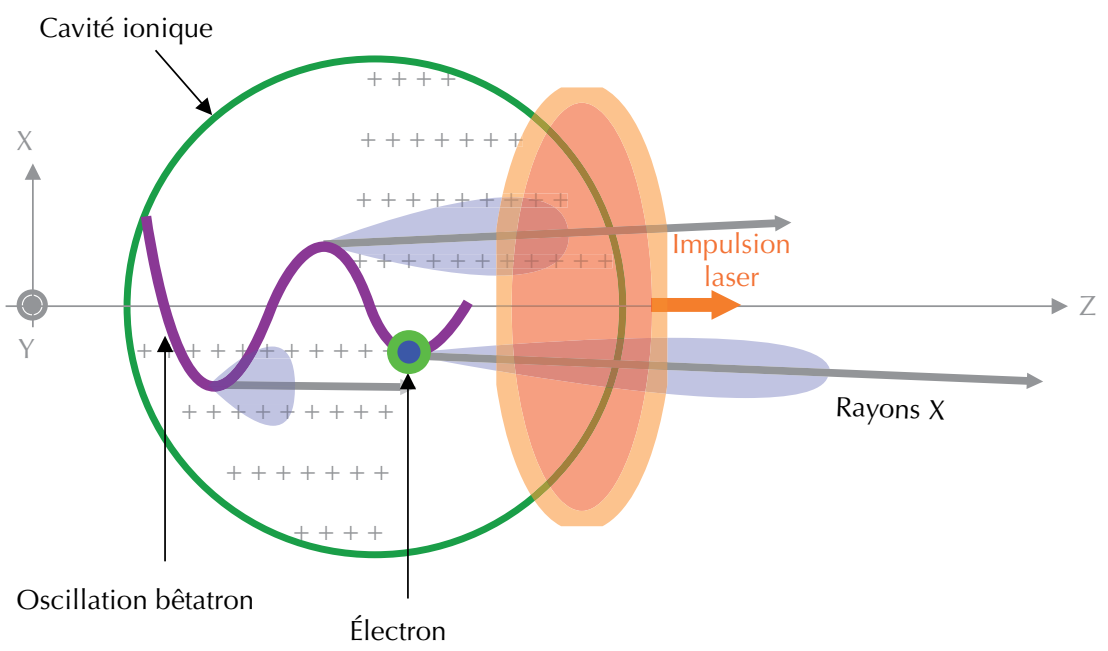

3. Rayonnement bêtatron. Les électrons injectés en dehors de l’axe laser oscillent dans le champ électrique transverse et émettent du rayonnement $X$ bêtatron. Le faisceau $X$ ainsi produit dans la direction du laser est ultra-bref et d'énergie atteignant quelques dizaines de keV pour des électrons de $300 \mathrm{MeV}$. 


\section{\〉>}

\section{Rayonnement Compton}

Si les électrons issus de l'accélérateur à plasma laser sont soumis au champ électromagnétique d'une impulsion laser intense contre-propagative, ils oscillent avec une longueur d'onde extrêmement courte, de l'ordre du micron, et émettent un rayonnement encore plus énergétique que dans le cas du rayonnement bêtatron (fig. 4). On peut voir ce mécanisme de deux manières différentes, mais équivalentes. Soit on considère le laser comme un onduleur (jouant le même rôle qu'un onduleur de synchrotron) de longueur
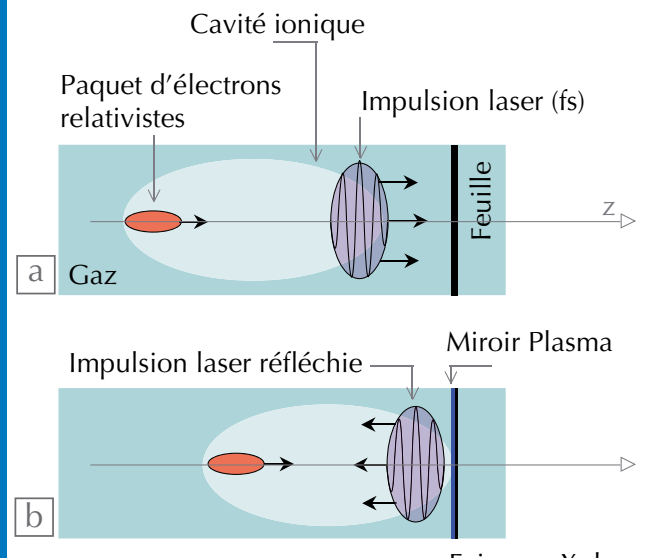

Faisceau X de haute énergie
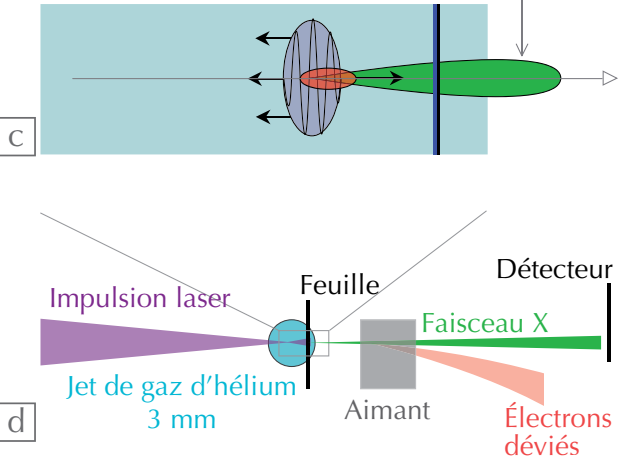

4. Diffusion Compton inverse. (a, b, c) Principe du phénomène. (d) Schéma de l'expérience.

(a) L'impulsion laser se propage dans un jet de gaz et crée une cavité ionique dans laquelle des électrons sont piégés et accélérés.

(b) L'impulsion laser, qui précède le paquet d'électrons, est ensuite réfléchie par une fine lame de verre placée après le jet de gaz. Elle entre alors en collision avec les électrons relativistes.

(c) Cette collision conduit à l'émission d'un faisceau intense de rayonnement Compton émis dans la direction de propagation du laser. La caractérisation de ce flash de rayonnement [8] a permis de mettre en évidence son spectre, qui s'étend du domaine $X$ à celui du rayonnement gamma, sa taille de source, qui est micrométrique, et sa divergence, qui est de l'ordre du degré. d'onde micrométrique, soit on voit ce mécanisme comme de la diffusion Thomson/Compton, processus par lequel un électron absorbe un ou plusieurs photons et émet un photon. Lorsque les directions de propagation du laser et des électrons sont opposées, l'énergie du rayonnement émis peut facilement atteindre la gamme de la centaine de $\mathrm{keV}$, et même s'étendre jusqu'à plusieurs $\mathrm{MeV}$ (rayonnement gamma). Du fait d'effets Doppler importants (d'une part sur la fréquence laser vue par les électrons et d'autre part sur le rayonnement qu'ils émettent mesuré dans le référentiel du laboratoire), l'énergie des photons émis est proportionnelle au produit de l'énergie des photons du laser par le carré de l'énergie des électrons. Ce mécanisme permet de produire des photons énergétiques en grand nombre. Dans l'expérience réalisée en 2012 au LOA [8], un schéma très simple a permis de réaliser une source 10000 fois plus brillante que les sources conventionnelles existantes dans cette gamme d'énergie.

\section{Applications et perspectives}

Les applications des faisceaux d'électrons issus des accélérateurs à plasma laser [7] couvrent de nombreux domaines.

(i) En science des matériaux, ils peuvent être utilisés pour l'inspection non destructive de la matière. Nous avons développé pour cette application une source gamma (s'étendant jusqu'à plusieurs dizaines de $\mathrm{MeV}$ ) utilisant le rayonnement de freinage produit lorsque les électrons traversent une cible (le convertisseur). Cette source permet l'imagerie d'hétérogénéités dans des objets denses avec une résolution spatiale inférieure à 50 microns.

(ii) En radiothérapie, les profils relativement homogènes de dose des faisceaux d'électrons de haute énergie $(\sim 200 \mathrm{MeV})$ pourraient permettre un traitement de certains cancers avec des performances plus intéressantes que les traitements actuels réalisés avec des sources X. Des expériences de radiobiologie ont permis de suivre les taux de survie et de réparation de cellules humaines irradiées par le faisceau d'électrons, et d'ouvrir ainsi une nouvelle approche pour l'étude des effets des rayonnements ionisants sur les tissus vivants.

Enfin, les nouvelles sources X, telles que les sources bêtatron et Compton, viennent compléter cette liste d'applications, à laquelle il faudra ajouter dans le futur le développement de lasers à électrons libres qui associeront accélérateurs laser plasma à des onduleurs, qu'ils soient classiques (c'est-à-dire comme ceux des synchrotrons, structures magnétiques périodiques), optiques ou plasma. Les sources $\mathrm{X}$ déjà produites sont extrêmement brillantes et ont permis d'obtenir des images par contraste de phase d'objets biologiques avec des résolutions de l'ordre du micromètre. Ultrabrèves (durée de l'ordre de la femtoseconde), elles autoriseront le suivi en temps réel des phénomènes ultrarapides pour les sciences du solide ou pour la biologie.

Concernant le futur de cette approche pour la physique des hautes énergies, les luminosités extrêmes exigées nécessiteront des développements importants, tant sur la technologie laser que sur la conception de schémas d'accélérateurs à multi-étages d'électrons et de positrons. Les puissances moyennes des faisceaux de particules requises (de l'ordre de quelques dizaines de MW) correspondraient, dans l'état actuel de nos connaissances et de la technologie laser, à des puissances électriques moyennes de l'ordre de quelques dizaines de GW. Le chemin à parcourir pour atteindre ces objectifs est donc pavé de nombreux défis qu'il nous faudra surmonter, ne rendant cette aventure qu'encore plus attrayante et excitante.

(1) La force pondéromotrice est une force non linéaire qu'une particule chargée subit dans un champ électromagnétique oscillant inhomogène.

(2) L'obtention du régime de la bulle nécessite d'avoir une impulsion laser de dimensions (longueur et largeur) inférieures à la longueur d'onde plasma.

\section{Bibliographie}

- L. di Ciaccio et $\mathrm{G}$. Hamel de Monchenault, Reflets de la physique $n^{\circ} 31$ (2012), pp. 17-19.

2• V. Malka et al., Science 298 (2002) 1596.

3• J. Faure et al., Nature 431 (2004) 541 ; C. Geddes et al., Nature 431 (2004) 538 ; S. Mangles et al., Nature 431 (2004) 535

4• J. Faure et al., Nature 444 (2006) 05393.

5• 0. Lundh et al., Nature Physics 7 (2011) 219.

$6 \bullet \mathrm{V}$. Malka, J. Faure et E. Lefebvre, La Recherche n³8 (février 2010).

\section{7• V. Malka et al., Nature Physics 4 (2008) 447.}

8• K. Ta Phuoc et al., Nature Photonics 6 (2012) 308 